\title{
Induction of heme oxygenase- 1 expression protects articular chondrocytes against cilostazol-induced cellular senescence
}

\author{
KANG MI KIM ${ }^{1}$, SI EUN PARK ${ }^{1}$, MI SUN LEE ${ }^{1}$, KOANHOI KIM $^{2}$ and YOUNG CHUL PARK ${ }^{1}$ \\ Departments of ${ }^{1}$ Microbiology and Immunology, and ${ }^{2}$ Pharmacology, \\ Pusan National University School of Medicine, Yangsan, Gyeongnam 626-870, Republic of Korea
}

Received June 6, 2014; Accepted August 25, 2014

DOI: $10.3892 /$ ijmm.2014.1918

\begin{abstract}
Chondrocyte senescence is associated with the aging and degeneration of cartilage, and eventually leads to joint destruction. The aim of this study was to elucidate the mechanisms responsible for the cytoprotective effects of heme oxygenase-1 (HO-1) on chondrocytes in cartilage. Chondrocyte senescence was induced using cilostazol and measured using a specific senescence-associated $\beta$-galactosidase (SA- $\beta$-gal) staining assay. Cilostazol altered the expression of type II collagen and $\beta$-catenin, which are phenotypic markers of the differentiation and dedifferentiation of chondrocytes. Cilostazol also significantly induced $\mathrm{HO}-1$ expression, and the induction of HO-1 expression was affected by a significant increase in reactive oxygen species (ROS) production caused by cilostazol treatment. Of note, pre-treatment with 3-morpholinosydnonimine hydrochloride (SIN-1), an inducer of HO-1 expression, markedly attenuated cilostazol-induced chondrocyte senescence, and thus, we examined whether HO-1 directly modulates chondrocyte senescence induced by cilostazol. The upregulation of HO-1 was found to suppress cilostazol-induced cellular senescence. In addition, the inhibition of HO-1 activity with the iron chelator, desferrioxamine (DFO), or HO-1 siRNA increased cilostazol-induced chondrocyte senescence. Based on these results, it can be concluded that HO-1 is associated with the suppression of chondrocyte senescence, and that the enforced overexpression of HO-1 protects chondrocytes against stress-induced senescence.
\end{abstract}

\section{Introduction}

Cellular senescence was originally described in normal cells that enter a state of permanent growth arrest after undergoing a finite number of divisions, resulting in progressive functional decline and eventual death. Senescent cells remain

Correspondence to: Dr Young Chul Park, Department of Microbiology and Immunology, Pusan National University School of Medicine, Busandaehak-ro 49, Yangsan, Gyeongnam 626-870, Republic of Korea

E-mail: ycpark@pusan.ac.kr

Key words: primary articular chondrocytes, cilostazol, cellular senescence, reactive oxygen species, heme oxygenase-1 metabolically active and are resistant to apoptosis induced by exposure to genotoxic stress (1). Furthermore, chondrocyte senescence plays important roles in aging and articular cartilage degeneration (2), causes a decline in chondrocyte numbers, and is important in the development and progression of osteoarthritis (OA) $(3,4)$. In fact, senescent chondrocytes accumulate with age and in the articular cartilages of patients with OA $(2,5,6)$. However, apoptotic chondrocyte death is not always a widespread phenomenon in the cartilage degeneration of OA or during cartilage aging (7-9).

Under physiological conditions, reactive oxygen species (ROS) are involved in the control of various aspects of biological processes, such as cell activation, proliferation and apoptotic death. Excessive ROS production induces oxidative stress and can damage extracellular matrix components and chondrocytes in articular cartilage, leading to cartilage destruction (10). Furthermore, age-related oxidative stress has been reported to render rat chondrocytes more susceptible to cell death mediated by oxidants (11). However, the factors that govern chondrocyte decisions regarding senescence and apoptotic death are incompletely understood.

Heme oxygenase-1 (HO-1) is a well-coordinated antioxidant enzyme that disrupts heme to form biliverdin, carbon monoxide and free iron. HO-1 is adaptively induced to remove the ROS produced by heavy metals, inflammatory cytokines and UV irradiation in a number of cell types (12-14), including chondrocytes $(15,16)$. In addition, it has been reported that HO-1 inhibits the production of interleukin (IL) $1 \beta$ and tumor necrosis factor (TNF)- $\alpha$, which participate in the pathogenesis of cartilage damage (17). Moreover, it has also been reported that the induction of HO-1 expression results in the downregulation of catabolic enzymes and inflammatory mediators in articular tissue, which suggests that $\mathrm{HO}-1$ is a component of the defense systems of articular cartilage $(18,19)$.

In the present study, we provide evidence that the induction of HO-1 expression protects articular chondrocytes against cellular senescence induced by cilostazol. This supports the view that HO-1 activity is associated with chondrocyte senescence and the pathogenesis of cartilage damage.

\section{Materials and methods}

Reagents. 3-Morpholinosydnonimine hydrochloride (SIN-1), protease inhibitor cocktail, desferrioxamine (DFO) and 
5-bromo-4-chloro-3-indolyl $\beta$-D-galactopyranoside (X-gal) were purchased from Sigma-Aldrich (St. Louis, MO, USA) Cilostazol (OPC-13013) was generously donated by Otsuka Pharmaceutical Co., Ltd. (Tokushima, Japan). FBS, Dulbecco's modified Eagle's medium (DMEM) and other culture reagents were purchased from HyClone Laboratories, Inc. (Logan, UT, USA). Anti-HO-1, type II collagen, $\beta$-catenin and $\beta$-actin antibodies were purchased from Santa Cruz Biotechnology, Inc. (Santa Cruz, CA, USA). Secondary horse radish peroxidase (HRP)-conjugated antibody and the enhanced chemiluminescence (ECL) western blotting kit were obtained from Amersham Pharmacia Biotech (Piscataway, NJ, USA).

Culture of articular chondrocytes. Articular chondrocytes for primary culture were isolated from slices of the knee joint cartilages of 5-week-old female Sprague-Dawley rats (Samtako Bio Korea, Osan, Korea), as previously described (20). Chondrocytes were isolated by enzymatic digestion for $1 \mathrm{~h}$ with $0.2 \%$ type II collagenase in DMEM. After collection by brief centrifugation, the cells were resuspended in DMEM supplemented with $10 \%$ heat-inactivated FBS and antibiotics $(50 \mathrm{U} / \mathrm{ml}$ of penicillin, $50 \mu \mathrm{g} / \mathrm{ml}$ of streptomycin) at $37^{\circ} \mathrm{C}$ in a $5 \% \mathrm{CO}_{2} /$ air atmosphere. The medium was replaced every 2 days, and the cells reached confluence after 4-5 days of culture, which was designated as passage 0 . Passage 1 cells were used for all experiments.

Evaluation of cell viability. Cell viability was determined using the trypan blue exclusion assay. Chondrocytes were plated at $1 \times 10^{5}$ cells per 6 -well plate, stabilized for $24 \mathrm{~h}$ and incubated for $48 \mathrm{~h}$ in the presence or absence of various concentrations of cilostazol in fresh DMEM medium. Following incubation, the cells were washed with phosphate-buffered saline (PBS) and viable cells were counted by trypan blue dye exclusion using a hemocytometer.

Senescence-associated $\beta$-galactosidase (SA- $\beta$-gal) staining assay. SA- $\beta$-gal staining assay was performed at $\mathrm{pH} 6.0$ as previously described with some modification $(20,21)$. Briefly, the cells were washed in PBS, fixed for 5 min (room temperature) in $0.2 \%$ glutaraldehyde $/ 2 \%$ formaldehyde, washed in PBS and incubated with SA- $\beta$-gal staining solution containing $1 \mathrm{mg} / \mathrm{ml} \mathrm{X-gal,} 40 \mathrm{mM}$ citrate/phosphate buffer ( $\mathrm{pH} \mathrm{6.0)}$, $5 \mathrm{mM}$ potassium ferrocyanide, $5 \mathrm{mM}$ potassium ferricyanide, $150 \mathrm{mM} \mathrm{NaCl}$ and $2 \mathrm{mM} \mathrm{MgCl}$, for $12 \mathrm{~h}$ at $37^{\circ} \mathrm{C}$. Degrees of senescence were obtained by expressing senescent cell numbers as percentages of total cell numbers.

Western blot analysis. Equivalent amounts $(20 \mu \mathrm{g})$ of total protein were loaded onto $12 \%$ SDS-polyacrylamide gels. The proteins were later transferred onto nitrocellulose membranes using an electroblotting apparatus (Bio-Rad, Richmond, CA, USA), and the membranes obtained were incubated with each primary antibody. The blots were washed with TBS-T and incubated with HRP-conjugated secondary anti-rabbit antibody. The membranes were then developed using the ECL reaction system and visualized using an LAS-3000 Luminescent Image Analyzer (Fujifilm, Tokyo, Japan). Image Gauge version 3.0 software was used to calculate changes in protein expression. $\beta$-actin was used as an internal control to confirm equal protein loading.
Measurement of intracellular ROS. Intracellular ROS was detected by monitoring changes in the fluorescence of the ROS-sensitive fluorophore, 2',7'-dichlorodihydrofluorescein diacetate $\left(\mathrm{H}_{2} \mathrm{DCF}-\mathrm{DA}\right)$, as previously described (22). Fluorescence was measured following incubation with $\mathrm{H}_{2}$ DCF-DA for 10 min using a Zeiss LSM 510 laser-scanning confocal microscope (Carl Zeiss, Inc., Göettingen, Germany). Absolute fluorescence intensities were determined using the same numbers of cells in randomly selected areas.

Preparation and transfection of cells with $\mathrm{HO}-1$ expression vector. HO-1 cDNA was a kindly donated by Dr Hun-Taeg Chung (Wonkwang University, Iksan, Korea). HO-1 was cloned into the pcDNA3 mammalian cell expression vector. Control cells were sham-transfected with the same plasmid excluding the HO-1 construct. Briefly, the chondrocytes were plated in 6-well plates at $5 \times 10^{5}$ cells/well, grown for $24 \mathrm{~h}$ and then transfected with $10 \mu \mathrm{g}$ of the HO-1 construct using Lipofectamine 2000 reagent (Invitrogen Life Technologies, Carlsbad, CA, USA). One day following transfection, the medium wase replated in fresh medium and the cells were treated with cilostazol.

Transfection of cells with HO-1 siRNA. A 21-nucleotide RNA with a 3'-dTdT overhang was synthesized by Dharmacon (Lafayette, CO, USA) to interfere exclusively with HO-1 mRNA expression. As a negative control, the same nucleotides were scrambled to form a non-genomic combination. The target of the AA-N19 mRNA was the HO-1 target sequence (5'-ACACUCAGCUUUCUGGUGGUU-3'). The chondrocytes were plated in 6 -well plates at $5 \times 10^{5}$ cells/well, grown for $24 \mathrm{~h}$ and then transfected with $30 \mathrm{nM}$ of siRNA per well using DharmaFECT ${ }^{\mathrm{TM}}$ DUOreagent, according to the manufacturer's instructions.

Statistics and reproducibility. The experiments were repeated at least 3 times. The results are expressed as the means \pm standard deviation (SD). The results for the experimental and control groups were tested for significant differences using the one-tailed Student's t-test. A value of $\mathrm{p}<0.05$ was considered to indicate a statistically significant difference.

\section{Results}

Cilostazol induces cellular senescence in primary articular chondrocytes. We first determined the effects of cilostazol on chondrocyte viability. Primary rat articular chondrocytes were isolated, maintained and treated with various concentrations $(2,10$, and $50 \mu \mathrm{M})$ of cilostazol for $48 \mathrm{~h}$ and then subjected to trypan blue dye exclusion assay. Cilostazol reduced cell proliferation, without having a significant toxic effect (Fig. 1A). The induction of cellular senescence was confirmed by an increase in the number of SA- $\beta$-gal-positive cells among the cilostazol-treated chondrocytes versus the untreated controls. Of note, cilostazol significantly increased the specific SA- $\beta$-gal staining of chondrocytes (Fig. 1B).

Next, to examine the effects of cilostazol on the maintenance of chondrocyte phenotypes, we examined the effects of cilostazol on the expression of type II collagen, a hallmark of chondrocytes. As shown in Fig. 2A, cilostazol significantly 

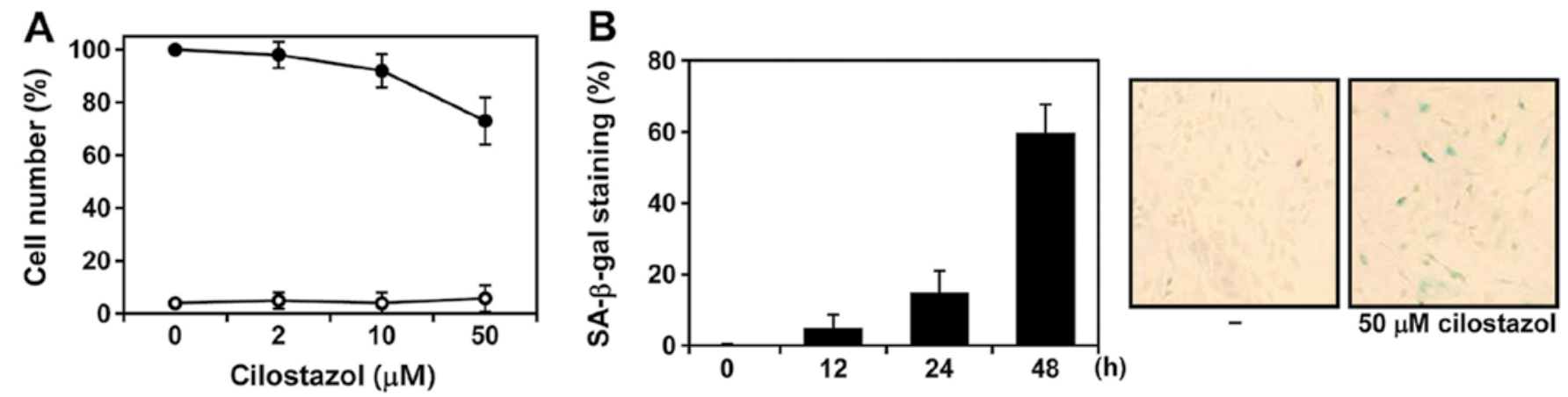

Figure 1. Effect of cilostazol on cellular senescence in chondrocytes. Rat articular chondrocytes were plated at $1 \times 10^{5}$ cells per 6 -well plate, incubated for $24 \mathrm{~h}$ and treated with cilostazol (2, 10, or $50 \mu \mathrm{M}$ for 12,24 , or $48 \mathrm{~h}$, as indicated). (A) Cells were assessed for viability using the trypan blue dye exclusion assay (live cells, $\bullet$; dead cells, O). Values are presented as percentages of the untreated control cells. (B) Chondrocyte senescence was confirmed using an senescence-associated $\beta$-galactosidase (SA- $\beta$-gal) activity assay. Percentages of SA- $\beta$-gal-positive cells were calculated from the numbers of blue cells per 200 cells in randomly selected areas. Representative images were obtained at a magnification of $\mathrm{x} 20$.



Figure 2. Effects of cilostazol on the expression of phenotypic markers in chondrocytes. Chondrocytes were treated with cilostazol (50 $\mu \mathrm{M}$ ) for the indicated periods of time. (A) Whole cell lysates were analyzed by western blot analysis using specific antibodies against type II collagen. $\beta$-actin was used as an internal control. (B) Cells were fixed and reacted with $\beta$-catenin antibody for immunofluorescence staining and examined using a confocal microscope. The right panels show superposition of $\beta$-catenin antibody and propidium iodide (PI) immunoreactivity (orange).

reduced the expression of type II collagen, which plays a crucial role in the regulation of the functions of chondrocyte via cell-matrix interactions. In addition, cilostazol stimulated the accumulation of $\beta$-catenin, a phenotypic marker of the differentiation and dedifferentiation of chondrocytes (Fig. 2B). These results suggest that cilostazol acts as an inducer of senescence in chondrocytes, as has been previously reported (20).

Cilostazol induces ROS generation and HO- 1 expression in chondrocytes. To determine whether the cellular senescence induced by cilostazol is associated with HO-1 expression, the cells were treated with various concentrations $(2,10$, and $50 \mu \mathrm{M}$ ) of cilostazol for $24 \mathrm{~h}$ and $\mathrm{HO}-1$ expression was analyzed by western blot analysis. It was found that the level of HO-1 expression was significantly increased by cilostazol (Fig. 3A). Subsequently, we examined whether the cilostazol-induced HO-1 expression is dependent on intracellular ROS generation in chondrocytes. As shown in Fig. 3B, a significant increase in DCF fluorescence was observed at 24 and $48 \mathrm{~h}$ after treating the cells with cilostazol. However, at early time points $(<12 \mathrm{~h})$, ROS generation was not detectable. These results reveal that cilostazol induces the expression of HO-1 by enhancing ROS generation in articular chondrocytes.

HO-1 induction inhibits cilostazol-induced cellular senescence in chondrocytes. In order to investigate the effects 
A

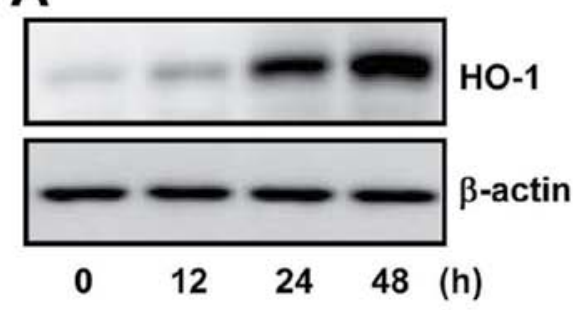

B

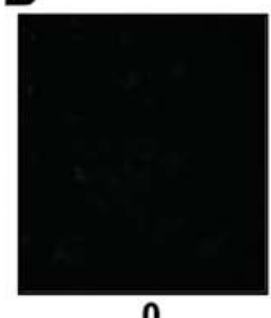

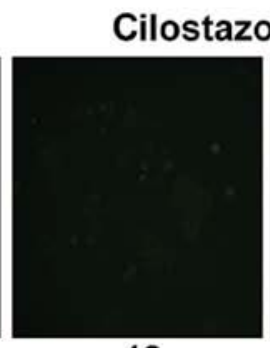

12

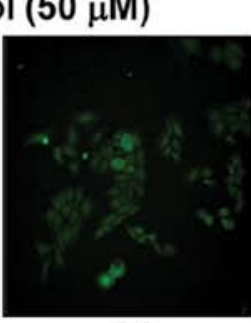

24

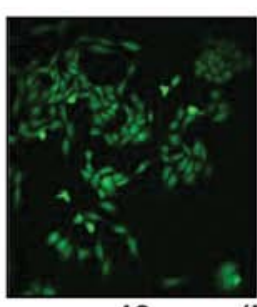

48

Figure 3. Effect of cilostazol on generation of reactive oxygen species (ROS) and heme oxygenase-1 (HO-1) expression in chondrocytes. Chondrocytes were incubated for the indicated periods of time in the absence or presence of $50 \mu \mathrm{M}$ cilostazol. (A) Whole cell lysates were analyzed by western blot analysis using a HO-1 antibody. (B) Intracellular ROS levels were determined using a confocal laser-scanning microscope.
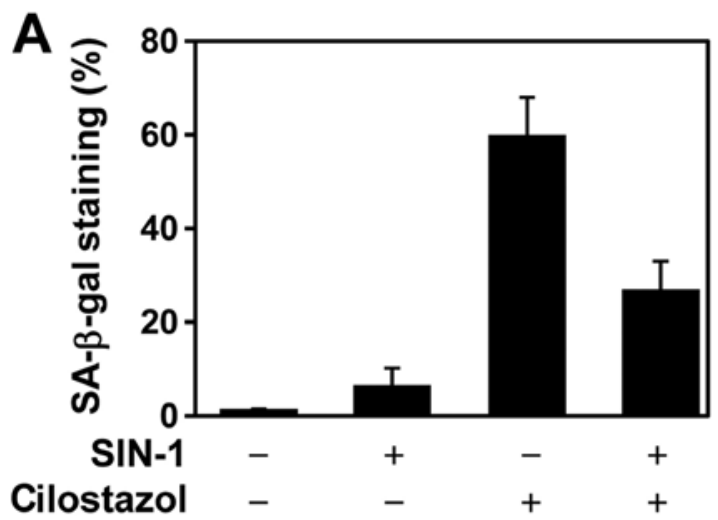

B
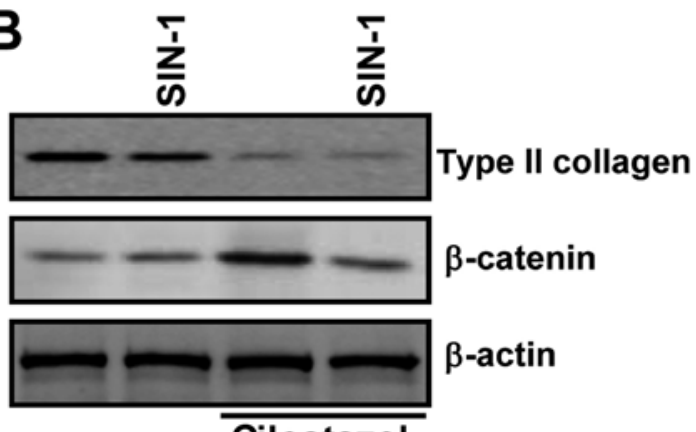

$\beta$-catenin

$\beta$-actin

Figure 4. Attenuation of stress-induced chondrocyte senescence by pre-treat ment with 3-morpholinosydnonimine hydrochloride (SIN-1). Chondrocytes were pre-treated with $200 \mu \mathrm{M}$ SIN-1 for $3 \mathrm{~h}$ and then stimulated with cilostazol $(50 \mu \mathrm{M})$ for $48 \mathrm{~h}$. (A) Chondrocyte senescence was determined using a senescence-associated $\beta$-galactosidase (SA- $\beta$-gal) activity assay. Values are the means \pm standard deviations (SD) of 3 independent experiments. (B) Whole cell lysates were analyzed by western blot analysis using specific antibody against $\beta$-catenin.

of HO-1 on cellular senescence, the chondrocytes were pre-treated with $100 \mu \mathrm{M}$ of SIN-1, an inducer of HO-1 expression, for $6 \mathrm{~h}$ prior to stimulation with cilostazol. Treatment with SIN-1 decreased the number of SA- $\beta$-gal-positive cells as compared with the chondrocytes treated with cilostazol only (Fig. 4A) and decreased the accumulation of $\beta$-catenin induced by cilostazol (Fig. 4B). Therefore, we examined whether HO-1 directly modulates cilostazol-induced cellular senescence by using the HO-1 expression vector. Of note, the overexpression of HO-1 was found to inhibit the cellular senes-

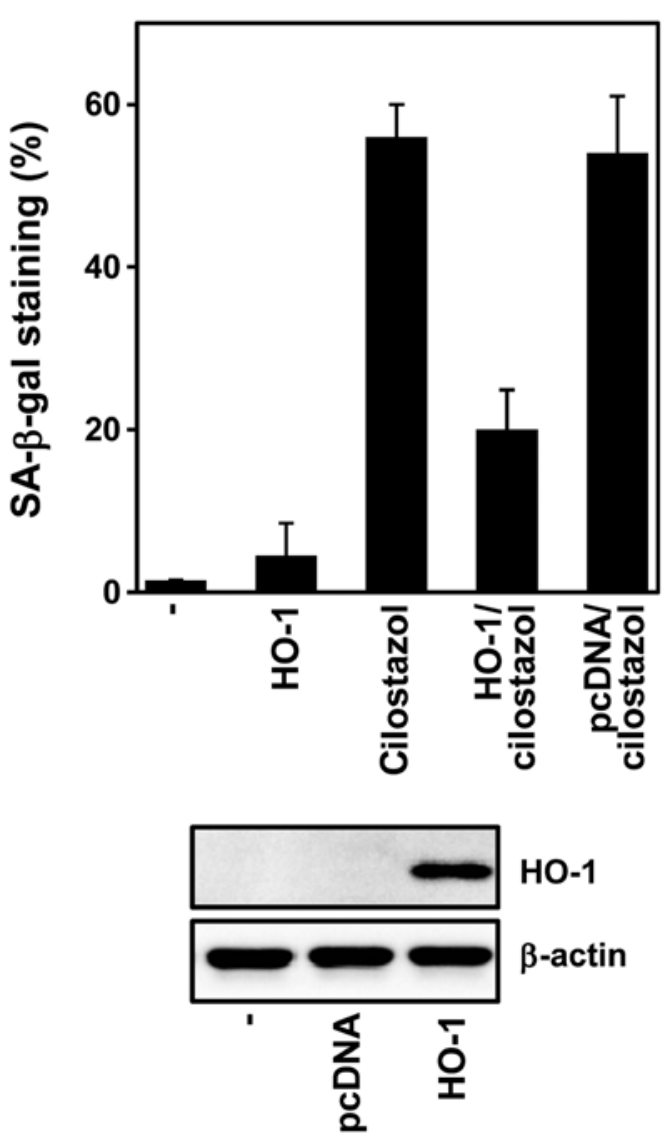

Figure 5. Effect of heme oxygenase-1 (HO-1) overexpression on the stress-induced senescence of chondrocytes. Chondrocytes were transfected with $\mathrm{HO}-1$ expression vector, incubated for $24 \mathrm{~h}$, treated with cilostazol $(50 \mu \mathrm{M})$ for $48 \mathrm{~h}$, and subjected to senescence-associated $\beta$-galactosidase (SA- $\beta$-gal) activity assay. Values are the means \pm standard deviations (SD) of 3 independent experiments. The gel demonstrates the induction of HO-1.

cence induced by cilostazol (Fig. 5). These results suggest that the upregulation of HO-1 has an anti-aging effect on chondrocyte senescence.

Inhibition of HO-1 enhances cilostazol-induced cellular senescence in chondrocytes. To investigate the effects of HO-1 activity on cilostazol-induced cellular senescence in chondrocytes, the iron chelator, DFO, was used to block HO-1 activity. The chondrocytes were pre-treated with $100 \mu \mathrm{M}$ DFO for $30 \mathrm{~min}$ and then incubated with cilostazol for $48 \mathrm{~h}$. At this 

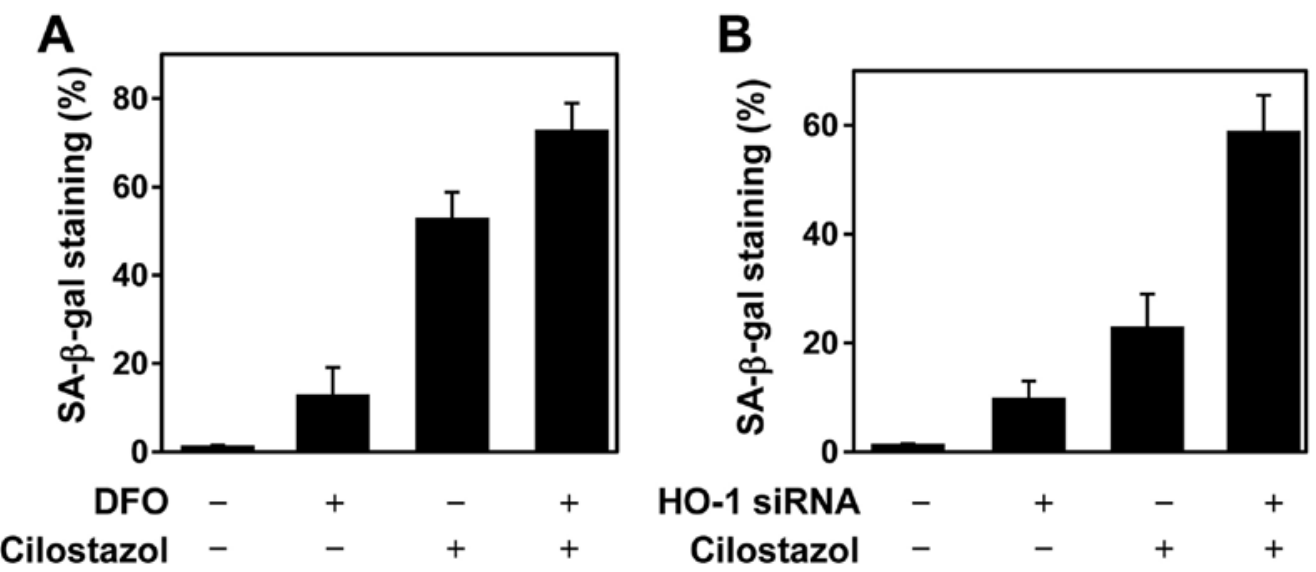

Figure 6. Effect of inhibition of heme oxygenase-1 (HO-1) on stress-induced chondrocyte senescence. (A) Chondrocytes were pre-treated with desferrioxamine (DFO) $(100 \mu \mathrm{M})$ for 30 min and then stimulated with cilostazol $(50 \mu \mathrm{M})$ for $48 \mathrm{~h}$. (B) Cells were transfected with siRNA in DharmaFECT ${ }^{\mathrm{TM}}$ DUO reagent to silence HO-1 gene expression, as described in 'Materials and methods'. Cells were treated with cilostazol for 24 h, and senescence-associated $\beta$-galactosidase (SA- $\beta$-gal) activity assay was performed. Whole cell lysates were analyzed by western blot analysis using a HO-1 antibody. Values are the means \pm standard deviations $(\mathrm{SD})$ of 3 independent experiments.

concentration DFO had no cytotoxic effect and abrogated HO activity (data not shown). As shown in Fig. 6A, the inhibition of $\mathrm{HO}$ activity significantly increased cilostazol-induced cellular senescence. To confirm this result, we used HO-1 siRNA to knockdown HO-1. HO-1 siRNA was found to effectively suppress HO-1 expression. In addition the cells transfected with HO-1 siRNA exhibited a greater degree of SA- $\beta$-gal staining than the control-transfected chondrocytes (Fig. 6B). These findings suggest that HO-1 activity is associated with chondrocyte senescence induced by cilostazol.

\section{Discussion}

Chondrocytes exist in avascular tissue in low oxygen environments. Nevertheless, chondrocytes produce ROS and have metabolisms that are adapted to anaerobic conditions and counteract abnormal levels of ROS produced by immune cells under pathological conditions. ROS can damage extracellular matrix components directly or indirectly by upregulating matrix metalloproteinases (MMPs), inducible nitric oxide synthase (iNOS), cyclooxygenase-2 (COX-2) and IL-6 genes (23-25). In addition, the age-related changes in the intracellular redox status of chondrocytes may be a major contributor to the increased apoptotic death and senescence of chondrocytes. However, the precise roles played by redox-related factors in cartilaginous tissue and chondrocytes remain largely unknown.

We recently reported that cilostazol accelerates cellular dedifferentiation and cellular senescence in primary rat articular chondrocytes (20). The present study demonstrates HO-1 exerts a protective effect against cellular senescence induced by cilostazol in articular chondrocytes. As shown in Fig. 3, cilostazol itself significantly induced ROS production and HO-1 expression in chondrocytes. Furthermore, the induction of HO-1 was found to be associated with cellular senescence in chondrocytes, and the upregulation of $\mathrm{HO}-1$ using SIN-1 or an HO-1 expression vector markedly suppressed cilostazol-induced cellular senescence (Figs. 4 and 5). In addition, the inhibition of HO-1 activity with the iron chelator,
DFO, or with HO-1 siRNA enhanced cilostazol-induced cellular senescence (Fig. 6). These results suggest that HO-1 protects chondrocytes against cilostazol-induced cellular senescence, and that HO-1 activity is associated with the chondrocyte senescence induced by oxidative stress. These results suggest that $\mathrm{HO}-1$ acts as an anti-aging factor during chondrocyte senescence.

HO-1 is considered to exert protective effects on cells and tissues exposed to cytotoxic conditions induced by oxidative stress in cartilage $(26,27)$. It has also been shown to have anti-inflammatory, anti-proliferative and anti-apoptotic effects due to its modulations of related gene expression or enzymatic activity $(28,29)$. HO-1 can act as a modulator of cellular homeostasis and regulate a variety of critical cellular processes in a number of different organisms. Therefore, HO-1 has been shown to be a positive regulator of chondrogenesis and chondrocyte differentiation (19). Importantly, HO-1 expression is upregulated by ROS and anti-inflammatory cytokines in chondrocytes and cartilage as well $(15,16,18)$. Previous studies have demonstrated that HO-1 significantly contributes to mechanisms that prevent the degeneration of human cartilage $(30,31)$. In addition, the upregulation of HO-1 has been shown to diminish osteoclast numbers in a model of rheumatoid arthritis, which suggests that HO-1 regulates osteoclastogenesis and consequently bone erosion in cartilage (31). However, although HO-1 expression is known to be induced to protect against the cartilage destruction elicited by oxidative stress, the role of HO-1 has not been thoroughly investigated. Thus, many researchers have focused on elucidating the regulation and functions of $\mathrm{HO}-1$ in chondrocytes and cartilage.

However, HO-1 may play a dual role in the pathology of tissue as high levels of HO-1 are frequently detected in pathological states $(32,33)$. Investigators have shown that the induction of HO-1 expresison by low dose sodium nitroprusside (SNP) protects human chondrocytes against apoptosis induced by high doses of this agent (34). Furthermore, age-related changes in the intracellular redox status of chondrocytes may be major contributors to the development of cellular senescence $(35,36)$, 
and thus, HO-1 may be a potential therapeutic target for diseases associated with chondrocyte senescence and apoptosis. For this reason, it is important to identify the precise role of HO-1 in the maintenance and death of chondrocytes in articular cartilage.

\section{Acknowledgements}

This study was supported by the Bio-Scientific Research Grant funded by Pusan National University (PNU, Bio-Scientific Research Grant) (PNU-2010-101-252).

\section{References}

1. Salminen A, Ojala $\mathbf{J}$ and Kaarniranta K: Apoptosis and aging: increased resistance to apoptosis enhances the aging process. Cell Mol Life Sci 68: 1021-1031, 2011.

2. Loeser RF: Aging and osteoarthritis: the role of chondrocyte senescence and aging changes in the cartilage matrix. Osteoarthritis Cartilage 17: 971-979, 2009.

3. Horton WE Jr, Feng L and Adams C: Chondrocyte apoptosis in development, aging and disease. Matrix Biol 17: 107-115, 1998

4. Kühn K, D'Lima DD, Hashimoto S and Lotz M: Cell death in cartilage. Osteoarthritis Cartilage 12: 1-16, 2004.

5. Martin JA and Buckwalter JA: Aging, articular cartilage chondrocyte senescence and osteoarthritis. Biogerontology 3 : 257-264, 2002.

6. Martin JA and Buckwalter JA: Telomere erosion and senescence in human articular cartilage chondrocytes. J Gerontol A Biol Sci Med Sci 56: B172-B179, 2001.

7. Aigner T, Hemmel M, Neureiter D, Gebhard PM, Zeiler G, Kirchner T and McKenna L: Apoptotic cell death is not a widespread phenomenon in normal aging and osteoarthritis human articular knee cartilage: a study of proliferation, programmed cell death (apoptosis), and viability of chondrocytes in normal and osteoarthritic human knee cartilage. Arthritis Rheum 44: 1304-1312, 2001

8. Hashimoto S, Ochs RL, Komiya S and Lotz M: Linkage of chondrocyte apoptosis and cartilage degradation in human osteoarthritis. Arthritis Rheum 41: 1632-1638, 1998.

9. Blanco FJ, Guitian R, Vázquez-Martul E, de Toro FJ and Galdo F: Osteoarthritis chondrocytes die by apoptosis. A possible pathway for osteoarthritis pathology. Arthritis Rheum 41: 284-289, 1998.

10. Jallali N, Ridha H, Thrasivoulou C, Underwood C, Butler PE and Cowen T: Vulnerability to ROS-induced cell death in ageing articular cartilage: the role of antioxidant enzyme activity. Osteoarthritis Cartilage 13: 614-622, 2005.

11. Muller M: Cellular senescence: molecular mechanisms, in vivo significance, and redox considerations. Antioxid Redox Signal 11: $59-98,2009$.

12. Taketani S, Kohno H, Yoshinaga T and Tokunaga R: The human 32-kDa stress protein induced by exposure to arsenite and cadmium ions is heme oxygenase. FEBS Lett 245: 173-176, 1989

13. Keyse SM and Tyrrell RM: Heme oxygenase is the major $32-\mathrm{kDa}$ stress protein induced in human skin fibroblast by UVA radiation, hydrogen peroxide, and sodium arsenite. Proc Natl Acad Sci USA 86: 99-103, 1989.

14. Durante W, Kroll MH, Christodoulides N, Peyton KJ and Schafer AI: Nitric oxide induces heme oxygenase-1 gene expression and carbon monoxide production in vascular smooth muscle cells. Circ Res 80: 557-564, 1997.

15. Valvason C, Musacchio E, Pozzuoli A, Ramonda R, Aldegheri R and Punzi L: Influence of glucosamine sulphate on oxidative stress in human osteoarthritic chondrocytes: effects on HO-1, p22(Phox) and iNOS expression. Rheumatology (Oxford) 47: $31-35,2008$
16. Kim KM, Song JD, Chung HT and Park YC: Protein kinase CK2 mediates peroxynitrite-induced heme oxygenase-1 expression in articular chondrocytes. Int J Mol Med 29: 1039-1044, 2012.

17. Eyre D: Collagen of articular cartilage. Arthritis Res 4: 30-35, 2002.

18. Henrotin YE, Bruckner P and Pujol JP: The role of reactive oxygen species in homeostasis and degradation of cartilage. Osteoarthritis Cartilage 11: 747-755, 2003.

19. Guillén M, Megias J, Gomar F and Alcaraz M: Haem oxygenase-1 regulates catabolic and anabolic processes in osteoarthritic chondrocytes. J Pathol 214: 515-522, 2008.

20. Kim KM, Kim JM, Yoo YH, Kim JI and Park YC: Cilostazol induces cellular senescence and confers resistance to etoposide-induced apoptosis in articular chondrocytes. Int J Mol Med 29: 619-624, 2012.

21. Dimri GP, Lee X, Basile G, Acosta M, Scott G, Roskelley C, Medrano EE, Linskens M, Rubelj I, Pereira-Smith O, et al: A biomarker that identifies senescent human cells in culture and in aging skin in vivo. Proc Natl Acad Sci USA 92: 9363-9367, 1995.

22. Lee SY, Jo HJ, Kim KM, Song JD, Chung HT and Park YC: Concurrent expression of heme oxygenase-1 and p53 in human retinal pigment epithelial cell line. Biochem Biophy Res Commun 365: 870-874, 2008.

23. Vincenti MP, Coon CI, Lee O and Brinckerhoff CE: Regulation of collagenase gene expression by IL-1 beta requires transcriptional and post-transcriptional mechanisms. Nucleic Acids Res 22: 4818-4827, 1994.

24. Pelletier JP, McCollum R, DiBattista J, Loose LD, Cloutier JM and Martel-Pelletier J: Regulation of human normal and osteoarthritic chondrocyte interleukin-1 receptor by antirheumatic drugs. Arthritis Rheum 36: 1517-1527, 1993.

25. Goldring MB: Osteoarthritis and cartilage: the role of cytokines. Curr Rheumatol Rep 2: 459-465, 2000

26. Wilks A: Heme oxygenase: evolution, structure, and mechanism. Antioxid Redox Signal 4: 603-614, 2002.

27. Otterbein LE and Choi AM: Heme oxygenase: colors of defense against cellular stress. Am J Physiol Lung Cell Mol Physiol 279: L1029-L1037, 2000.

28. Duckers HJ, Boehm M, True AL, Yet S-F, San H, Park JL, Webb RC, Lee M-E, Nabel GJ and Nabel EG: Heme oxygenase-1 protects against vascular constriction and proliferation. Nat Med 7: 693-698, 2001

29. Pae HO, Choi BM, Oh GS, Lee MS, Ryu DG, Rhew HY, Kim YM and Chung HT: Roles of heme oxygenase-1 in the antiproliferative and antiapoptotic effects of nitric oxide on Jurkat T cells Mol Pharmacol 66: 122-128, 2004.

30. Fernández P, Guillén MI, Gomar F and Alcaraz MJ: Expression of heme oxygenase-1 and regulation by cytokines in human osteoarthritic chondrocytes. Biochem Pharmacol 66: 2049-2052, 2003.

31. Zwerina J, Tzima S, Hayer S, Redlich K, Hoffmann O, Hanslik-Schnabel B, Smolen JS, Kollias G and Schett G: Heme oxygenase $1(\mathrm{HO}-1)$ regulates osteoclastogenesis and bone resorption. FASEB J 19: 2011-2013, 2005.

32. Bauer M and Bauer I: Heme oxygenase-1: redox regulation and role in the hepatic response to oxidative stress. Antioxid Redox Signal 4: 749-758, 2002

33. Otterbein LE, Kolls JK, Mantell LL, Cook JL, Alam J and Choi AM: Exogenous administration of heme oxygenase-1 by gene transfer provides protection against hyperoxia-induced lung injury. J Clin Invest 103: 1047-1054, 1999.

34. Kim HA, Lee KB and Bae S-C: The mechanism of low-concentration sodium nitroprusside-mediated protection of chondrocyte death. Arthritis Res Ther 7: R526-R535, 2005.

35. Carlo MD Jr and Loeser RF: Increased oxidative stress with aging reduces chondrocyte survival: correlation with intracellular glutathione levels. Arthritis Rheum 48: 3419-3430, 2003.

36. Murray MM, Zurakowski D and Vrahas MS: The death of articular chondrocytes after intra-articular fracture in humans. J Trauma 56: 128-131, 2004. 